\title{
Comparison of acoustic quantification and Doppler echocardiography in assessment of left ventricular diastolic variables
}

Adrian Chenzbraun, Fausto J Pinto, Shawn Popylisen, Ingela Schnittger, Richard L Popp

\begin{abstract}
Objective-To assess the haemodynamic correlations of the waveforms of left ventricular area change obtained by automated boundary detection with newly developed acoustic quantification technology.

Design-The timing of events in the cardiac cycle was identified on the waveform automated boundary detection and was correlated with the corresponding timing derived from pulsed wave Doppler flow velocity traces of the mitral valve and left ventricular outflow tract. The amounts of area change during the rapid filling phase and during atrial contraction were correlated with the time-velocity integrals of early and late diastolic ventricular filling obtained from Doppler tracings of the mitral inflow.

Setting-A university medical school echocardiography laboratory.

Subjects-16 healthy volunteers and 19 patients referred for echocardiographic studies.
\end{abstract}

Results-A significant correlation was found between the methods for measurement of the time from the $R$ wave to mitral valve opening $(r=0.72, p<0.01)$, isovolumic relaxation time $(r=0.62, p<$ $0.01)$, and ejection time $(r=0.54, p<$ $0.01)$. The change of total area that occurred during rapid filling and atrial filling phases measured from the acoustic waveform correlated with the time-velocity integrals of the early and late diastolic mitral valve inflow velocity derived from Doppler echocardiography $(r=0.60$ and $r=0.80$, respectively).

Conclusion-The waveform of left ventricular area obtained by the automated boundary detection technique identifies the phases of the cardiac cycle and correlates with Doppler values of left ventricular diastolic function. Therefore, this new method of automated boundary detection has potential uses in the assessment of left ventricular diastolic function.

(Br Heart f 1993;70:448-456)
Division of Cardiovascular Medicine, Standford University School of Medicine, Stanford, California

A Chenzbraun

F J Pinto

$S$ Popylisen

I Schnittger

R L Popp

Correspondence to:

Richard L Popp MD,

Division of Cardiology, Stanford University Sch,
of Medicine, Stanford, CA 94305 .

Accepted for publication 27 April 1993

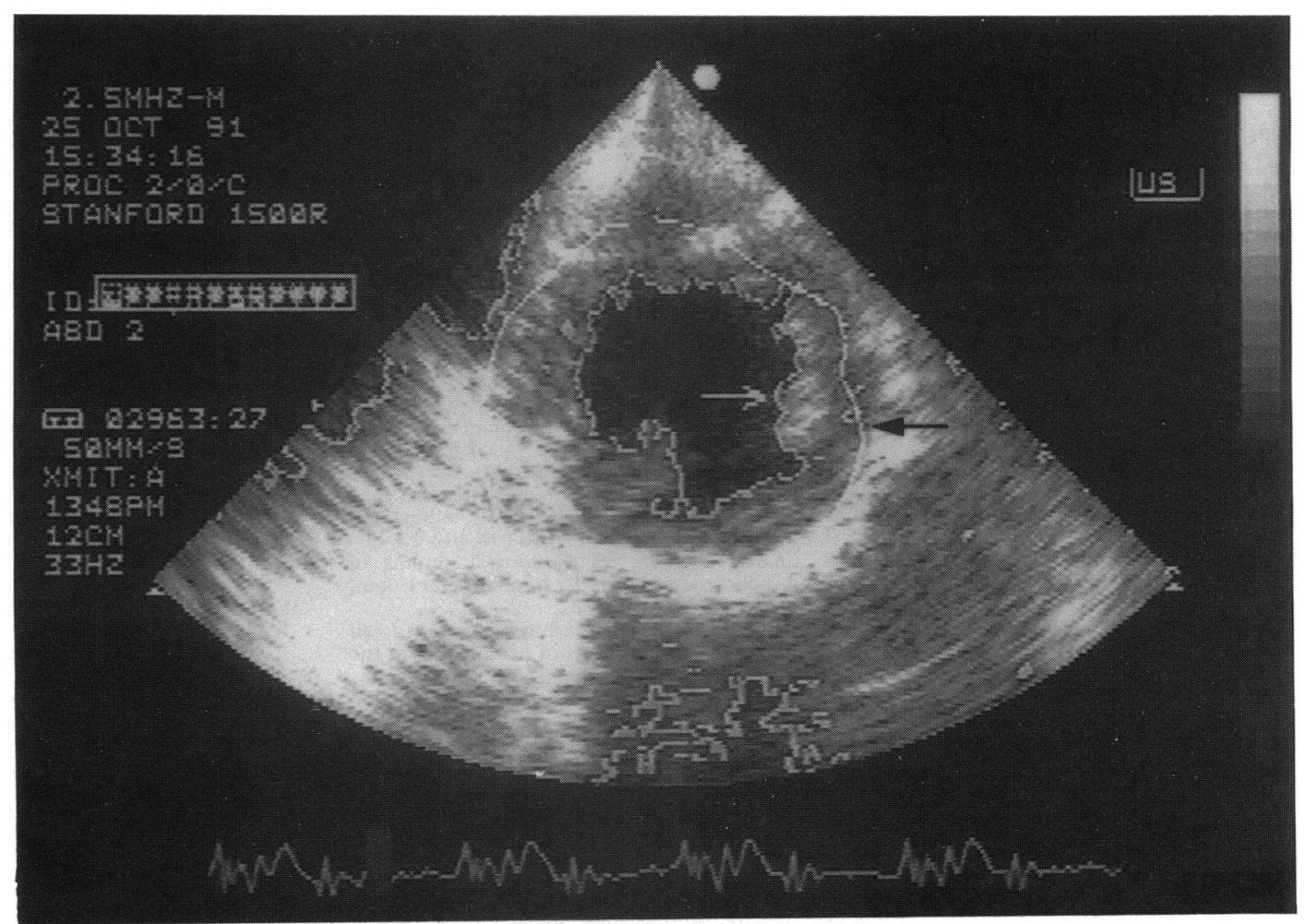

Figure 1 Real time parasternal short axis view of the left ventricle at papillary muscle level with the automated border detection method. The ventricular cavity is included in a manually traced region of interest (black arrow). $A$ thin bright line (white arrow) identifies the blood endocardial surface. 
Figure 2 Left. Waveforms display. Upper panel area change waveform $\left(\mathrm{cm}^{2}\right)$.

Lower panel: dA/dt waveform $\left(\mathrm{cm}^{2} \mathrm{~s}\right)$. A, end diastole; $B$, beginning of ejection; $C$, end of ejection $D$, mitral valve opening: $E$, end of rapid filling;
$F$, beginning of atrial contraction. Right. Left ventricular volume waveform (upper trace) and aortic pressure waveform (lower trace) from the classic paper of

Wiggers and $\mathrm{Katz}^{3}$ (reproduced with permission).
A B

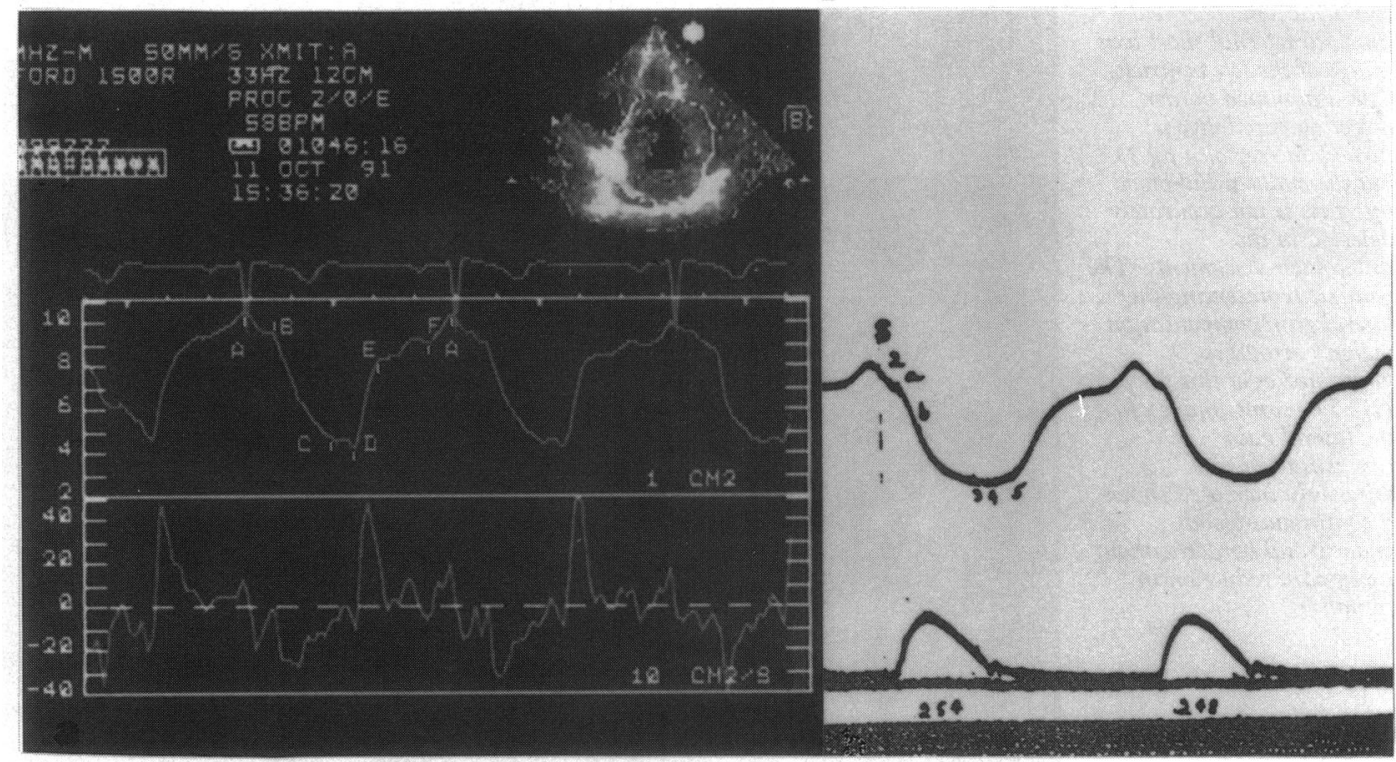

Non-invasive measurement of left ventricular volume throughout the cardiac cycle has many potential uses. The left ventricular volume curve is currently obtained predominantly from radionuclide ventriculography. Manual traces of echocardiographic images provide volumetric data comparable with those obtained by contrast ventriculography ${ }^{1}$ but data acquisition on sequential frames throughout the cardiac cycle is tedious and time consuming, thus precluding the routine use of this method. An acoustic technique with the ability to automatically identify, display, and track the endocardial-cavity interface has been developed recently and incorporated in commercially available ultrasonographs. The detection of the blood-tissue interface is based on ultrasonic backscatter technology. ${ }^{2}$ The endocardial-luminal border appears as a thin bright line and the area enclosed by it represents the instantaneous cross sectional area (fig. 1). A waveform representing the changing value of the area is displayed along with the cross sectional image allowing for on line area measurements throughout the cardiac cycle. This waveform is strikingly similar to the classic left ventricular volume curve obtained by physiologists (fig 2) ${ }^{3}$ Conversion of direct measurement of the cross sectional area of the ventricular chamber to left ventricular volume requires application of formulae not yet validated for this method. A display of the rate of area change versus time $(\mathrm{dA} / \mathrm{dt})$ is also available. The purpose of this study was to analyse the waveform of the left ventricular area change obtained by the ultrasonic technique with automated border detection, define its values in normal subjects, and evaluate it as a potential tool for the assessment of left ventricular diastolic function.

\section{Patients and methods}

STUDY POPULATION

Thirty five subjects (18 men, 17 women) aged 18 to 80 (mean (SD): 42(16) years in sinus rhythm and with good quality cross sectional and Doppler echocardiographic studies were enrolled. Sixteen of them were healthy volunteers with no cardiovascular history and normal physical examination. The remaining subjects were referred for echocardiographic examination because of mitral valve prolapse (four), assessment of left ventricular function (four), assessment of murmur (three), assessment after radiofrequency ablation of accessory conduction tissue bundles (three), coronary artery disease (two), prior stroke (one), suspected cardiomyopathy (one), and suspected left ventricular hypertrophy (one). Patients with dyskinetic segments by cross sectional echocardiography or with more than mild mitral or aortic regurgitation by colour Doppler flow study were excluded. All subjects gave informed consent for the protocol, which was approved by the Committee for the Protection of Human Subjects at the Stanford University Medical Center.

\section{ECHOCARDIOGRAPHIC STUDIES}

A complete echocardiographic study, including cross sectional pulsed wave, and Doppler colour flow imaging was performed according to standard techniques with a HewlettPackard Sonos 1500 ultrasonograph and 2.5 or $3.5 \mathrm{MHz}$ transducers.

\section{ACOUSTIC IMAGING}

An optimal standard ultrasound image of the left ventricle was obtained in the parasternal short axis view at the papillary muscle level and further improved if necessary by reducing the depth of field and the sector width. The acoustic quantification system was then activated and a prototype lateral gain control circuit was used to increase the signal in ultrasonic lines in selected areas of interest at the cavity-wall interface, to enhance endocardial echoes (fig 3). The automated border detection capability was then turned on and the system displayed a border following the detected cavity wall interface. The accuracy of the endocardial tracking was assessed by 
Figure 3 Lateral gain compensation. (A) real time parasternal short axis image of the left ventricle. The automated border detection capability is turned on (see also fig 1) but the endocardial blood interface is not accurately detected in the anterolateral segments. The triangle representing the lateral gain compensation region (arrow) is positioned over this area. (B) The same image after the lateral gain compensation was selectively increased shows a continuous blood endocardial border without significant reduction in signal.

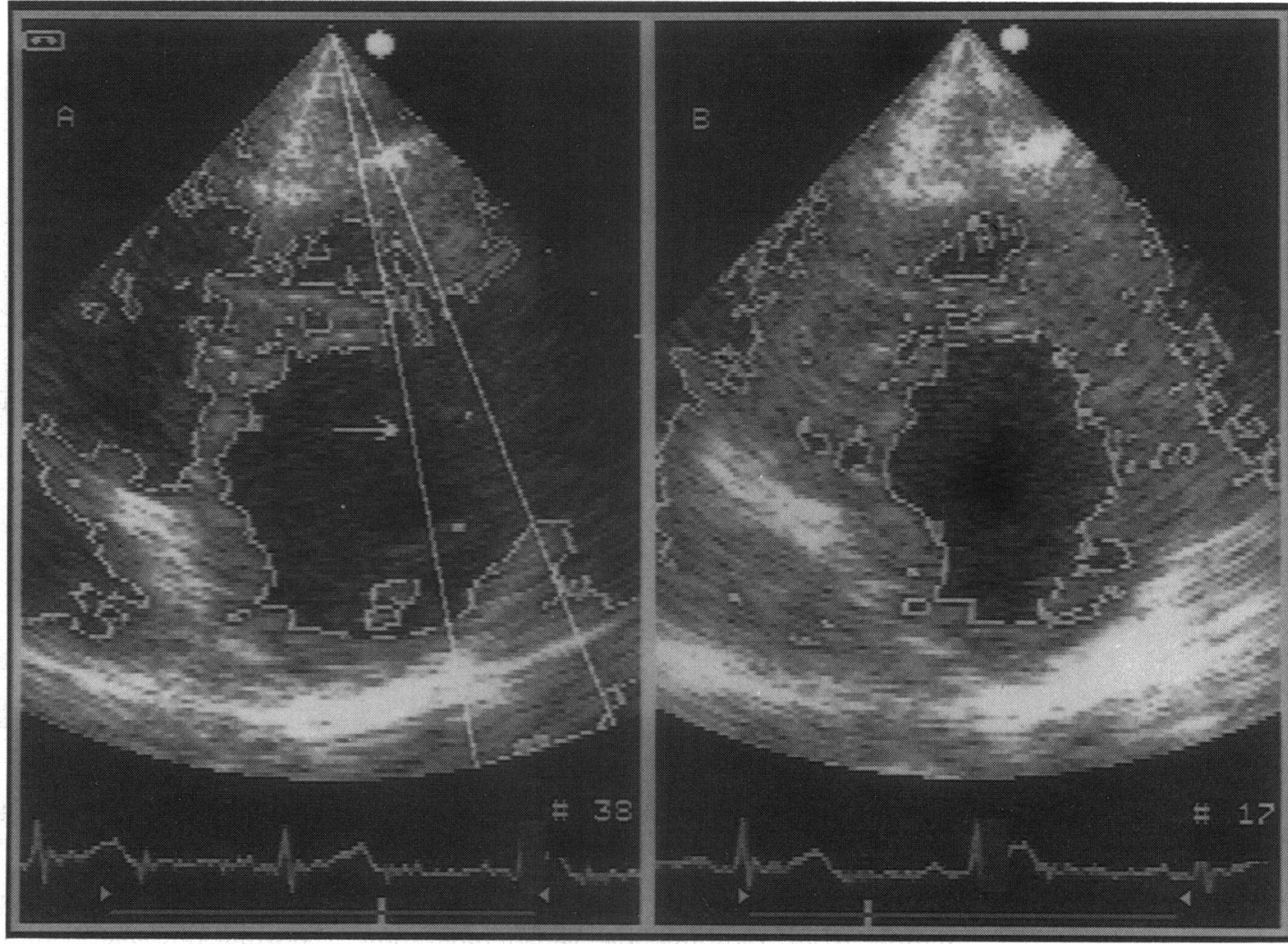

observation of its contour and movement and by turning this border on and off to compare it visually with the cavity-endocardial interface. A study was considered satisfactory if at least two thirds of the endocardial contour was correctly traced. A region of interest for area calculation was then manually traced within the myocardium so that the ventricular cross-sectional area was included throughout the cardiac cycle (fig 1). The waveforms of left ventricular area change and the rate of area change $(\mathrm{dA} / \mathrm{dt})$ were then displayed along with the electrocardiogram and the concurrent cross sectional image (fig. 2).

\section{Doppler recordings}

After imaging was completed, a spectral Doppler recording of the mitral inflow was recorded at a paper speed of 50 and 100 $\mathrm{mm} / \mathrm{s}$ from the apical four chamber view with the pulsed wave sample volume positioned at the tips of the mitral valve leaflets. ${ }^{4}$ The sample volume was then placed in the left ventricular outflow tract, so a recording of both the left ventricular outflow tract and the mitral inflow were obtained for measurements of ejection time and isovolumic relaxation time. ${ }^{5}$ All studies were recorded on 0.5 inch super VHS videotape.

\section{Waveform analysis}

Off line analysis was performed in all studies with the calibration and measurement system built into the ultrasonograph. The following points were defined on the area waveform (fig 2): point $A$, at the peak of the $\mathrm{R}$ wave on the electrocardiogram, was considered to represent end diastole; points $B$ and $C$, at the beginning and at the end of the abrupt downslope of the curve, were considered to mark the beginning and the end of the left ventricular ejection period; point $D$, at the beginning of the abrupt upslope of the curve, was considered to represent the opening of the mitral valve and the beginning of the rapid filling period; point $E$, at the end of this segment, was considered to represent the completion of the rapid filling period; point $F$, at the beginning of the upslope segment near end diastole, was considered to represent the beginning of area change due to atrial contraction. End diastolic and end-systolic areas were taken at points $\mathrm{A}$ and $\mathrm{C}$, and the areas at the end of the rapid filling period and at the beginning of the atrial contraction were taken at points $\mathrm{E}$ and $\mathrm{F}$, respectively. The decrease in the cross sectional area of the left ventricle from end diastole to end systole was defined as fractional area change and calculated as:

\section{Fractional area change $=$}

$(A$ area $-C$ area $) / A$ area

The amount of the total area change that occurred at the end of the rapid filling period was defined as rapid filling fractional area change and was calculated as:

Rapid filling fractional area change $=$

$$
(E \text { area }-C \text { areal }(A \text { area }-C \text { area })
$$

The amount of the total area change that occurred during atrial contraction was defined as atrial filling fractional area change and was calculated as:

Atrial filling fractional area change $=$

$$
\text { ( } A \text { area }-F \text { area }) /(A \text { area }-C \text { area })
$$

The amount of total area change that occurred during the slow filling period was defined as slow filling fractional area change and was calculated as:

Slow filling fractional area change $=$

$(F$ area $-E$ area $) /(A$ area $-C$ area $)$ 
The following time intervals were measured from the waveform: ejection time, between points $B$ and $C$; isovolumic relaxation time, between points $\mathrm{C}$ and $\mathrm{D}$; rapid filling time, between points $D$ and $E$; time from the $R$ wave to the opening of the mitral valve, between the peak of the $R$ wave and point $D$. The rapid filling slope was measured as the mean slope of the DE segment. The first upward peak in diastole on the $\mathrm{dA} / \mathrm{dt}$ waveform, was considered to represent the peak rate of area change during rapid ventricular filling. The time to peak rate of area change was measured as the time interval from endsystole (point $\mathrm{C}$ on the area waveform) to this point. All subjects were in sinus rhythm and values for three cardiac cycles were averaged for each measurement.

\section{DOPPLER ANALYSIS}

The Doppler analysis was performed by one of the investigators who was blinded to the waveform measurements. The video tape was replayed and the following variables of left ventricular diastolic function were measured from the mitral inflow Doppler flow velocity tracings from the Doppler analysis package of the echocardiographic system (fig 4): peak early diastolic velocity $(E)$, peak late velocity (A), E/A ratio, acceleration slope and pressure half time of early mitral flow, time velocity integral during rapid filling $\left(\mathrm{E}_{\text {intg }}\right)$, time velocity integral during atrial contraction ( $A_{\text {intg }}$ and total time velocity integral of the ventricular filling $\left(\mathrm{TF}_{\text {ints }}\right)$. The rapid filling and atrial filling contributions to the total filling were calculated as $\mathrm{E}_{\text {intg }} / \mathrm{TF}_{\text {ints }}$ and $A_{\text {intg }} / T_{\text {inty }}$, respectively. An E/A ratio $<1$ was considered diagnostic of abnormal relaxation. The slow filling contribution to total filling was not measured owing to expected difficulties in quantifying this low velocity portion of the mitral inflow. The following time intervals were measured: ejection time, isovolumic relaxation time, rapid filling time, and time from the peak of the $R$ wave to the opening of the mitral valve.

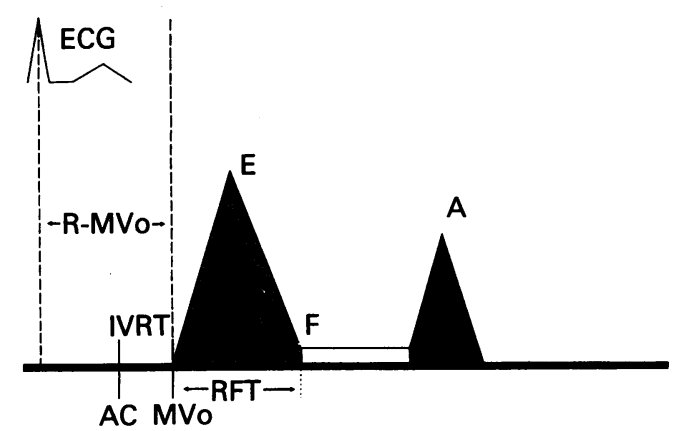

Figure 4 Schematic diagram showing the Doppler trace analysis. $A$, peak velocity during atrial contraction; $A C$, aortic valve closure click; $E$, peak velocity during early filling; ECG, electrocardiogram; IVRT, isovolumic filling; ECG, electrocardiogram; IVRT, isovolumic
relaxation time.; $M V O$, mitral valve opening click; $R F T$, rapid filling time; $R-M V o$, time from the peak of the $R$ wave to the opening of the mitral valve. Hatched areas: time velocity integral of early and late filling. When the trace included a distinct slow filling period, a line was trace included a distinct slow filling period, a line was time and time velocity integral measurements.

\section{DOPPLER ACOUSTIC QUANTIFICATION} WAVEFORM CORRELATIONS

The ejection time, the isovolumic relaxation time, the rapid filling time, and the time from the $R$ wave to mitral valve opening as measured on the acoustic waveform were compared with the corresponding intervals on the Doppler tracing. The ratio of rapid filling fractional area change to atrial filling fractional area change, the rapid filling slope, the peak rate of area change during rapid filling, and the time to peak area change in the waveform from the acoustic technique were correlated with Doppler indices of diastolic function: E/A ratio, rapid filling contribution, atrial filling contribution, rapid filling contribution/atrial filling contribution ratio, acceleration slope, and pressure half time.

\section{DATA ANALYSIS}

Data were expressed as mean (SD). We compared indices of ventricular filling from Doppler and acoustic quantification by two approaches. Firstly, simple regression was used to assess the linear association between the two methods. Secondly, the method of Bland and Altman was used to assess the difference in measurements made by the two methods; both limits of agreement and confidence intervals (CIS) were calculated. ${ }^{6}$ Group differences were assessed by Student's $t$ test. Results were considered significant at $\mathrm{p} \leqslant 0.05$.

\section{REPRODUCIBILITY OF MEASUREMENTS}

To assess intraobserver variability one investigator reanalysed the videotaped studies. End diastolic and end systolic areas were remeasured and the area change at the end of rapid filling was recalculated in 12 patients. Linear regression analysis and absolute differences between repeat off line measurements were used to define reproducibility. ${ }^{7}$ Good reproducibility was obtained for measurements of end diastolic areas $(r=0.99$, mean error $=$ $4 \%)$, end systolic areas $(r=0.98$, mean error $=9 \%)$ and rapid filling fractional area change $(r=0.83$, mean error $=6 \%)$. Ten randomly selected, previously videotaped studies were also assessed by a second investigator to obtain interobserver reproducibility of the same measurements. Good reproducibility was found for all the measurements: end diastolic area $(r=0.98$, mean error $=3 \%)$, end systolic area $(r=0.98$, mean error $=4 \%)$, and rapid filling fractional area change $(r=$ $0 \cdot 72$, mean error $=7 \%$ ).

\section{Results}

ECHOCARDIOGRAPHIC DOPPLER RESULTS

Doppler studies were within normal limits in 25 of the 35 subjects. One of them had mitral valve prolapse but the other 24 had normal cross sectional examinations. Twenty one of these 24 subjects (age $=34(4)$, range 29-42) were considered to represent a normal population in view of their negative history and physical examination whereas the remaining three had known heart disease (one 
Table 1 Systolic and diastolic values of the waveform from acoustic quantification of area in 21 normal subjects

\begin{tabular}{lc}
\hline EDA $\left(\mathrm{cm}^{2}\right)$ & $12.5(3)$ \\
ESA $\left(\mathrm{cm}^{2}\right)$ & $6.4(2)$ \\
FAC & $0.5(0.12)$ \\
RFFAC & $0.74(0.07)$ \\
AFFAC & $0.19(0.07)$ \\
RFFAC/AFFAC & $4.51(2)$ \\
SFFAC & $0.07(0.07)$ \\
T-PRAC $(\mathrm{ms})$ & $174(23)$ \\
PRAC $\left(\mathrm{cm}^{2} / \mathrm{s}\right)$ & $50(12)$ \\
RFS $\left(\mathrm{cm}^{2} / \mathrm{s}\right)$ & $35.4(10)$ \\
\hline
\end{tabular}

${ }^{\star}$ Data from 13 subjects; †Data from 15 subjects. EDA, end diasolic area; ESA, end systolic area; FAC, fractional area change; RFFAC, rapid filling fractional area change; AFFAC, atrial filling fractional area change; SFFAC, slow filling fractional area change; T-PRAC, time to peak rate of area change during rapid filling; PRAC, peak rate of area change during rapid filling; RFS, slope of rapid filling segment.

had coronary artery disease and two had arrhythmia). A pattern of abnormal relaxation by Doppler criteria $(\mathrm{E} / \mathrm{A}<1)$ was found in 10 of these 35 subjects. Systolic dysfunction was present in only three of them by cross sectional examination.

\section{MORPHOLOGY OF WAVEFORMS REPRESENTING AREA CHANGE}

Figure 2 shows representative waveform and the reference points for events in the cardiac cycle. Table 1 shows the values found in the normal population. Short axis left ventricular end diastolic and end systolic area averaged 12.5 (3) $\mathrm{cm}^{2}$ and $6.4(2) \mathrm{cm}^{2}$, respectively, with a fractional area change of $0.5(0 \cdot 12)$. Time intervals on the acoustic waveform were compared with the Doppler trace in the subjects in whom they were available from both methods. Table 2 shows the correlation coefficients. Significant correlations were found for isovolumic relaxation time $(r=0.62, \mathrm{p}<$ 0.01 ), time from the peak of the $R$ wave to the opening of the mitral valve $(r=0.72, \mathrm{p}<$ $0.01)$, rapid filling time $(r=0.44, \mathrm{p}<0.01)$, and ejection time $(r=0.54, \mathrm{p}<0.01)$.

\section{DIASTOLIC PORTION OF THE WAVEFORM}

A good correlation was found in the 35 subjects between the rapid filling fractional area change, atrial filling fractional area change and the ratio of rapid filling fractional area change to atrial filling fractional area change

Table 2 Correlation of time intervals measured on the waveform from acoustic quantification and the Doppler trace

\begin{tabular}{lllll}
\hline $\begin{array}{l}\text { Time interval } \\
\text { (ms) }\end{array}$ & Waveform & $\begin{array}{l}\text { Doppler } \\
\text { trace }\end{array}$ & $r$ & $p$ Value \\
\hline ET (n=26) & $267(33)$ & $287(24)$ & 0.54 & $<0.01$ \\
IVRT (n=24) & $88(16)$ & $90(19)$ & 0.62 & $<0.01$ \\
RFT (n=33) & $129(32)$ & $225(52)$ & 0.44 & $<0.01$ \\
RMVo (n=34) & $459(50)$ & $444(45)$ & 0.72 & $<0.01$ \\
\hline
\end{tabular}

$A Q$, acoustic quantification; ET, ejection time; IVRT, isovolumic relaxation time; RFT, rapid filling time; $R-M V o$, time from peak of $R$ to the mitral valve opening.

Table 3 Correlations between the rapid filling and the atrial contribution to the total ventricular filling as determined from the waveform from acoustic quantification and the Doppler traces

\begin{tabular}{lllll}
\hline Diastolic index & $\begin{array}{l}\text { AQ } \\
\text { waveform }\end{array}$ & Doppler & $r$ & $p$ Values \\
\hline Early filling & $0.67(0.13)$ & $0.59(0.1)$ & 0.59 & $<0.01$ \\
Atrial filling & $0.25(0.13)$ & $0.28(0.12)$ & 0.80 & $<0.01$ \\
Early/atrial filling & $3.6(0.2)$ & $2.56(1.5)$ & 0.75 & $<0.01$ \\
\hline
\end{tabular}

$\mathrm{AQ}$ acoustic quantification by the new acoustic technique and the corresponding Doppler rapid filling contribution, atrial filling contribution, ratio of rapid filling contribution to atrial filling contribution and the $\mathrm{E} / \mathrm{A}$ ratio (table 3, fig 5). The CIs for the mean differences between the acoustic technique and Doppler measurements were relatively narrow. There was, however, considerable scatter in the differences between the absolute values given by the two methods for the left ventricular filling indices (fig 5). A striking contrast in the morphology of the acoustic waveform was evident between the 10 subjects with and the 25 subjects without abnormal relaxation by Doppler criteria (fig 6). Patients with abnormal relaxation had significantly lower rapid filling fractional area change, higher atrial filling fractional area change, lower slope of area change during the rapid filling period, and a lower peak rate of area change (table 4). No correlation was found between the acoustic variables and the acceleration slope or the pressure half time measured by Doppler.

\section{Discussion}

\section{ACOUSTIC QUANTIFICATION}

Previous attempts at automated detection of the left ventricular endocardial border on standard cross sectional echocardiographic images required the intervention of an operator to define and monitor the endocardial border. ${ }^{8}$ The integrated backscattered ultrasound energy from blood within the ventricular cavity is sufficiently low compared with the signal from the myocardium that the interface between blood and muscle can be detected with the new acoustic quantification system. By integrating the information for all lines, on line reconstruction and real time tracking and display of the endocardial edge is provided allowing for measurements of left ventricular cross sectional area throughout the cardiac cycle. ${ }^{9}$ The accuracy of this method compared with manual edge tracing for area measurements has been validated by previous studies..$^{90}$ Measurements of left ventricular cross sectional areas have been used as the basis for ventricular volume calculations based on several geometric models. ${ }^{11} \mathrm{~A}$ method has been proposed by which the end diastolic and end systolic cross sectional areas at papillary muscle level are the only values needed for calculation of volume. ${ }^{1}$ In view of these data, it may be assumed that changes in the left ventricular cross sectional area throughout the cardiac cycle will correlate with changes in ventricular volumes as long as akinetic or dyskinetic segments are not present. The differences in the shape of abnormal ventricles and the change in shape from diastole to systole in the beating heart make the conversion of the area to volume less exact than the use of area values for the purposes of this study. The main advantages of acoustic quantification technology over manual area tracing are the continuous, on line nature of the data supplied and the convenience of the automatic border detection. 
Figure 5 Scatterplots of left ventricular filling indices measured by acoustic quantification and Doppler echocardiography. (A) Rapid filling phase contribution to filling (B) atrial contribution to filling, (C) ratio of rapid and atrial contribution to filling. Upper panels: linear regression, lower panels: plot of the

difference between the $A Q$ filling indices and the corresponding Doppler indices on the $y$ axis and their mean on the $x$ axis. horizontal solid line, ideal zero difference; horizontal dashed line, mean difference; $\square$ limits of agreement at mean (2SD); $A Q$, acoustic quantification; $A F C$, atrial contribution to filling by Doppler; AFFAC, atrial filling fractional area change; $R F C$, rapid filling contribution by Doppler; RFFAC, rapid filling fractional area change.
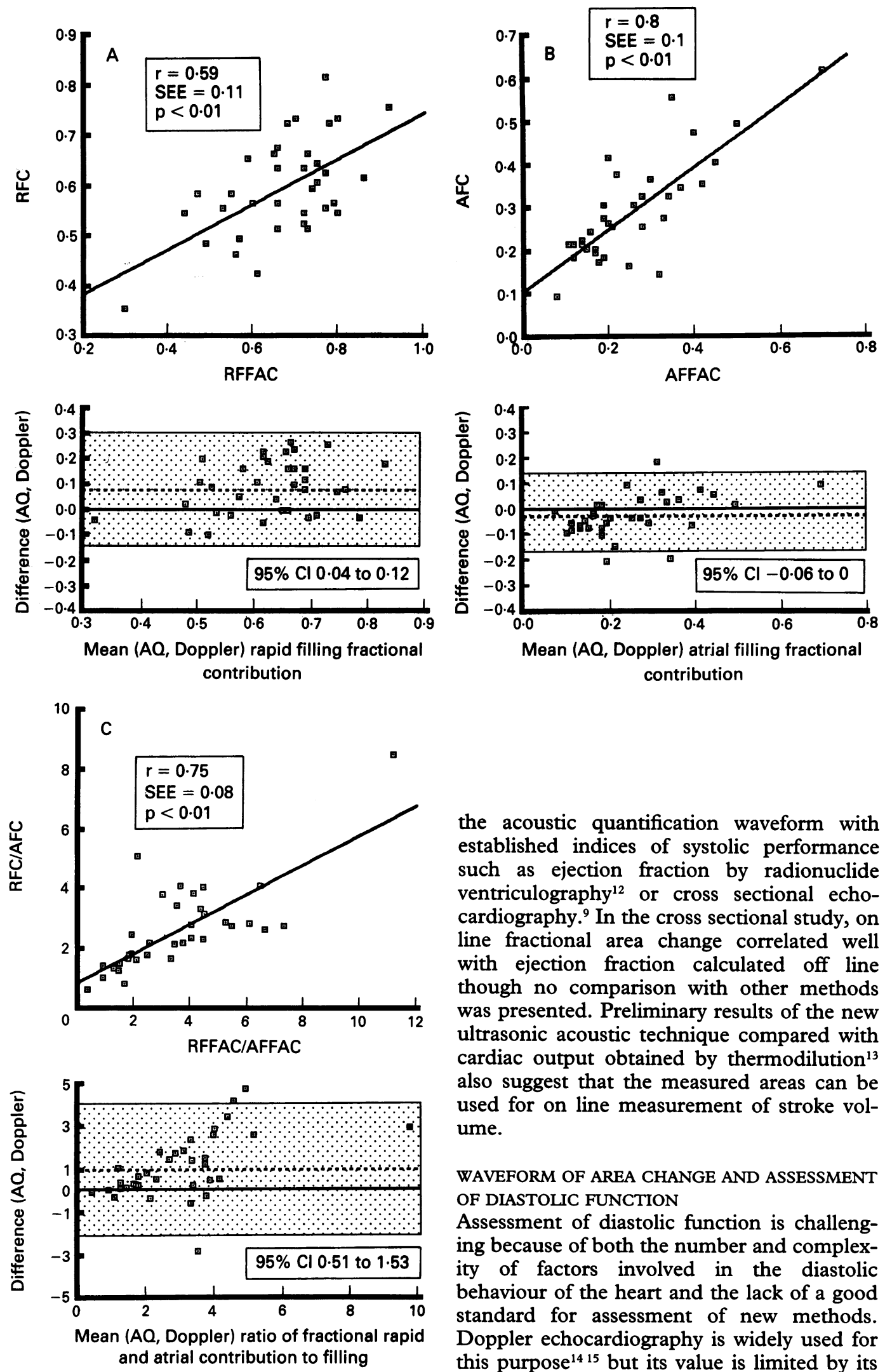

This kind of analysis would be extremely difficult and tedious with manual tracing techniques. The available waveform follows the area changes during the cardiac cycle, and thus reflects the dynamics of ventricular filling and emptying. Previous studies have shown the potential of acoustic measurements for assessing systolic function by comparing the fractional area change provided by the acoustic quantification waveform with established indices of systolic performance such as ejection fraction by radionuclide ventriculography ${ }^{12}$ or cross sectional echocardiography. ${ }^{9}$ In the cross sectional study, on line fractional area change correlated well with ejection fraction calculated off line though no comparison with other methods was presented. Preliminary results of the new ultrasonic acoustic technique compared with cardiac output obtained by thermodilution ${ }^{13}$ also suggest that the measured areas can be used for on line measurement of stroke volume.

WAVEFORM OF AREA CHANGE AND ASSESSMENT OF DIASTOLIC FUNCTION

Assessment of diastolic function is challenging because of both the number and complexity of factors involved in the diastolic behaviour of the heart and the lack of a good standard for assessment of new methods. Doppler echocardiography is widely used for this purpose $e^{14}$ but its value is limited by its dependence on changes in cardiac pressures and loading conditions. ${ }^{41617}$ Radionuclide ventriculography ${ }^{18}$ or ultrafast computed tomography ${ }^{19}$ provide accurate information on filling patterns but they are expensive, time consuming and not suitable for serial examination. As discussed, the area waveform derived from acoustic quantification may permit the analysis of the ventricular filling pattern by a technique that is relatively simple, available, easy to perform serially, and which provides information on a beat to beat basis. 
Figure 6 Morphology of the waveform from acoustic quantification. (A) subject with a normal Doppler pattern, (B) patient with an abnormal relaxation pattern. Upper panels: Doppler trace, lower panels: waveforms from acoustic quantification. Arrow: atrial component of ventricular filling. In patient (B) the diastolic filling shows a prominent atrial component, which is of higher amplitude and with a more abrupt upslope than in the trace of the normal subject $(A)$ whose filling is represented primarily by the early rapid filling segment.
A

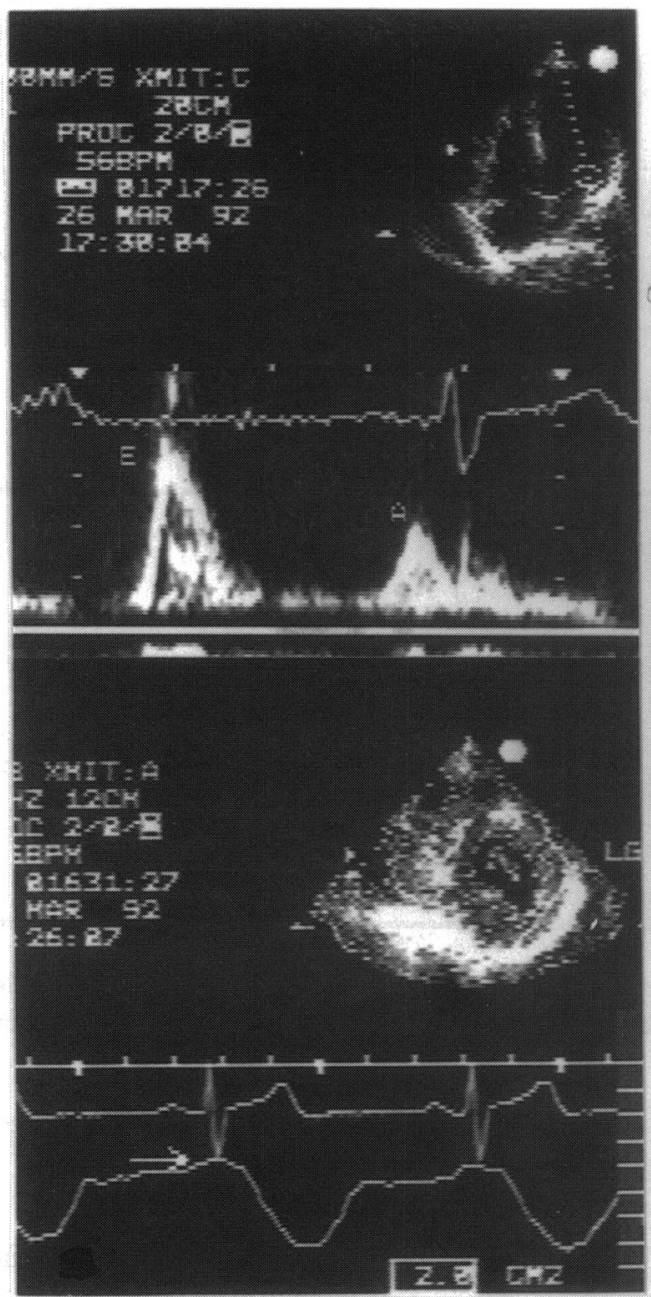

B

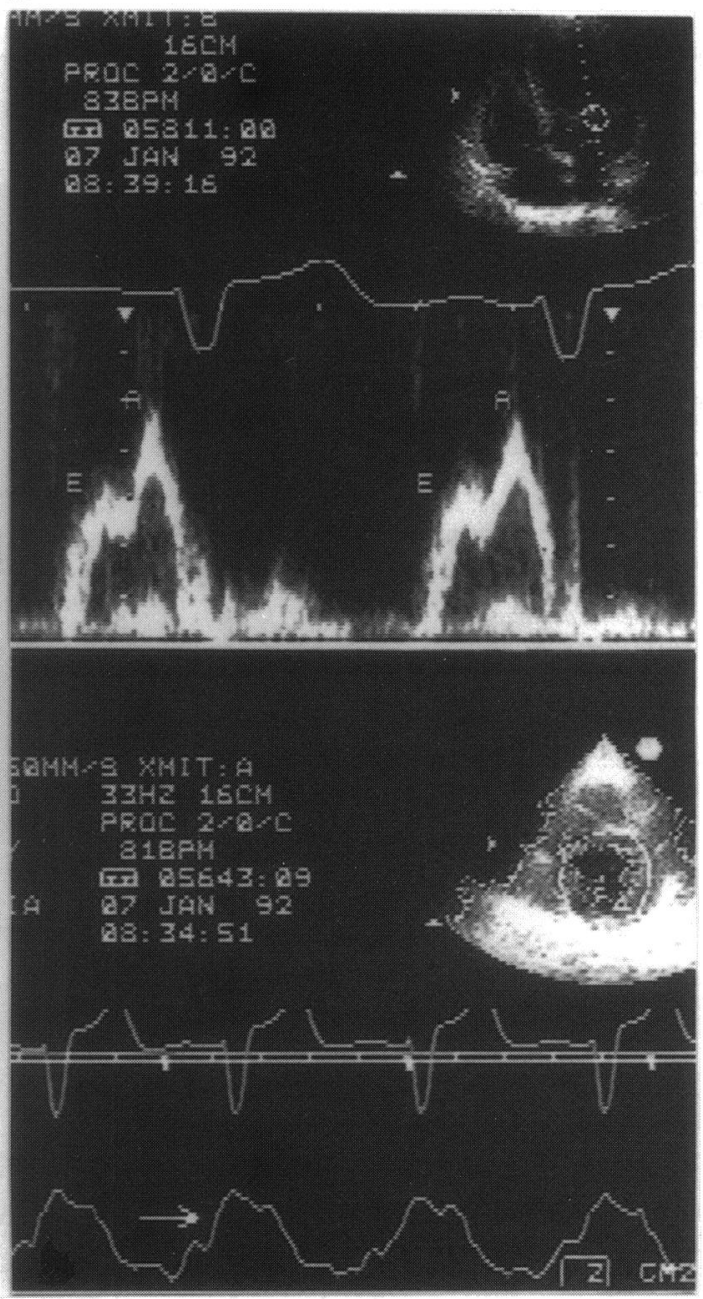

PRESENT STUDY

Acoustic quantification waveform morphology

The shape of the acoustic quantification waveform and the time correlations with the Doppler trace shows that this area waveform accurately identifies the phases of the cardiac cycle (fig 7). The weakest correlation between methods was found for the rapid filling time. We postulate that a significant decrease in the filling rate may occur before the mitral flow velocity signal approaches the baseline. Therefore, Doppler measurements of the rapid filling time may be longer than those obtained by the acoustic quantification measurements. The fact that the rapid filling period values measured on the acoustic quantification waveform were consistently shorter than those measured on the Doppler trace is

Table 4 Diastolic indices of the waveform from acoustic quantification in subjects with normal and abnormal quantification
relaxation

\begin{tabular}{lccc}
\hline Diastolic index & $\begin{array}{l}\text { Normal } \\
\text { relaxation } \\
(n=25)\end{array}$ & $\begin{array}{l}\text { Abnormal } \\
\text { relaxation } \\
(n=10)\end{array}$ & p Value \\
\hline RFFAC & 0.74 & 0.51 & $<0.01$ \\
AFFAC & 0.19 & 0.40 & $<0.01$ \\
RFFAC/AFFAC & $4 \cdot 44$ & 1.43 & $<0.01$ \\
PRAC $\left(\mathrm{cm}^{2} / \mathrm{s}\right)$ & $50.40^{\star}$ & $33.50 \dagger$ & $<0.01$ \\
RFS $\left(\mathrm{cm}^{2} / \mathrm{s}\right)$ & 35.40 & 23.30 & $<0.01$ \\
\hline
\end{tabular}

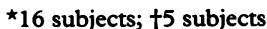

Abbreviations as in table 1 . consistent with this explanation. (fig 7, table 2).

\section{NORMAL VALUES}

Any new technique requires reference values to be defined in a normal population before it can be used in clinical studies. The values we obtained for end diastolic area, end systolic area, and fractional area change in our normal subjects (table 1) are similar to those reported by Klein et $a l^{20}$ using the same technique and may be used to assess serially ventricular areas and systolic performance.

\section{ACOUSTIC OUANTIFICATION WAVEFORM AND}

DIASTOLIC FUNCTION

The finding in this normal population that $74 \%$ of the changes in total area is completed at the end of the early rapid filling segment of the diastolic portion of the waveform is consistent with earlier data obtained with radionuclide ventriculography showing that $60 \%-80 \%$ of the ventricular filling occurs at the end of the rapid filling period in normal subjects. ${ }^{21} 22$ Also, the time to peak area change found in this study is similar to the normal values for time to peak filling rate as measured by radionuclide ventriculography..$^{23}$ These data suggest that the acoustic area waveform reflects the volume-waveform and could be used to assess diastolic function. 


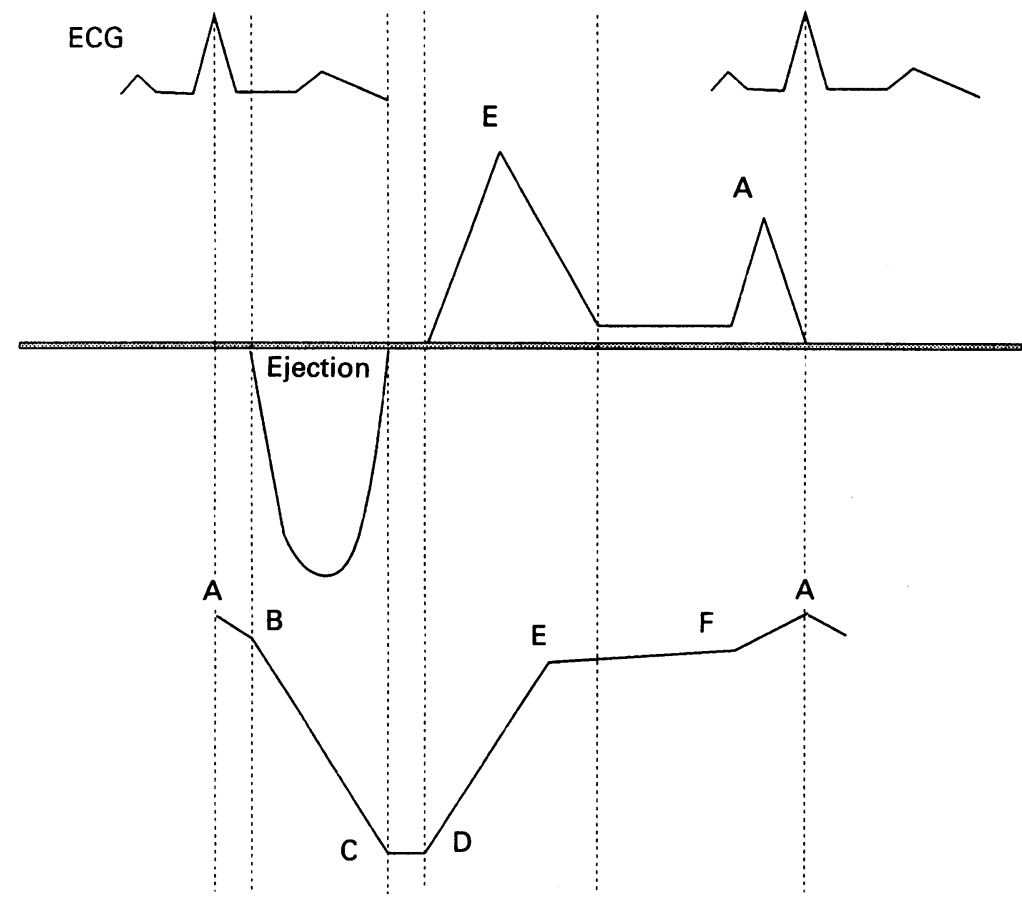

Figure 7 Schematic drawing of the time correspondence for diastolic events between the Doppler trace (upper trace) and the waveform from acoustic quantification (lower trace). The rapid filling time by Doppler was consistently longer than by the waveform from acoustic quantification. Abbreviations as in fig 2 and 4.
Doppler derived values of diastolic function for comparison with the acoustic quantification waveform. This represents only one widely used method with well known limitations. There were no haemodynamic measurements to compare with, as none of the subjects had clinical indications for cardiac catheterisation. Specifically, defining abnormal relaxation as Doppler patterns with an $\mathrm{E} / \mathrm{A}$ ratio $<1$ is an oversimplification. This criterion is widely accepted in clinical practice and probably is a reasonable definition in the absence of hypovolaemia or acute vasodilatation. Another potential limitation is related to the waveform itself. Although good inter and intra observer variabilities were found for the measured values it was also appreciated that occasionally there was difficulty in identifying specific points if the waveform which was either too smooth or too contaminated by artefacts. Theoretically, using the $\mathrm{dA} / \mathrm{dt}$ derivative waveform to identify inflexion points on the area waveform could be a potential solution of this problem. The quality of the $\mathrm{dA} / \mathrm{dt}$ waveform, however, was not always adequate for the purpose. This should change in the future with improvement in the detection and processing algorithms. The acoustic measurements in this study were areas and area derived values. Volume measurements are now calculated on commercially available versions of this equipment. As the volumes are obtained from algorithms that are based on the measured areas, significant discrepancies are not, however, expected. Also, data on validation of the volume measurements compared with contrast ventriculography are still lacking. Finally, the volumetric change gauged by the new acoustic method is different from that gauged from the velocity patterns of the Doppler method. The effective area of the mitral valve is continously changing during the phases of ventricular filling, so the relation of the velocity of flow to volumetric flow is complex. One would not expect extremely high correlation coefficients between these two methods even with simultaneous recordings. Perhaps one strength of the new method of acoustic quantification will be to complement the Doppler echocardiographic assessment of diastolic filling of the left ventricle. Further studies including patients with a range of well defined diastolic abnormalities and a range of loading conditions are required to understand better the place of this new technology in the evaluation of diastolic dysfunction.

Dr Chenzbraun was supported in part by the Israeli Heiden Fellowship, Jerusalem, Israel and by the American Physician Fellowship, Brookline, MA, USA. Dr Pinto is the recipient of a fellowship award from Fundação Calouste Gilbenkian, Lisbon, Portugal.

Due to limitations in the software, the Doppler and the acoustic traces could not be recorded simultaneously; also, we had no way to quantify or to control the variable time delay between the electrocardiogram and the acoustic waveform (ranging from minimal to one frame time $+20 \mathrm{~ms}$ ). These factors probably account for lack of higher correlation for time intervals of the cardiac cycle between the two techniques. In our study we used
1 Parisi AF, Moynihan PF, Feldman CL, Folland ED. Approaches to determination of left ventricular volume Approaches to determination of left ventricular volume
and ejection fraction by real time two-dimensional and ejection fraction by real time two-dim

2 Perez JE, Miller JG. Ultrasonic backscatter tissue characterization in cardiac diagnosis. Clin Cardiol 1991;14: terization.

3 Wiggers CJ, Katz LN. The contour of the different volume curves under different conditions. Am $\mathcal{f}$ Physiol 1922;58:439-75. 
4 Appleton CP, Hatle L, Popp RL. Relation of transmitral flow velocity patterns to left ventricular diastolic function: new insights from a combined hemodynamic and tion: new insights from a combined hemodynamic and 1988;12:426-39.

5 Sasson Z, Hatle L, Appleton CP, Jewett M, Alderman EL, Popp RL. Intraventricular flow during isovolumic relaxation: description and characteristics by Dopple echocardiography. F Am Coll Cardiol 1987;10:539-47.

6 Bland MJ, Altman DG. Statistical method for assessing agreement between two methods of clinical measurement. Lancet 1986;1:307-10.

7 Pietro DA, Voelkel AG, Ray BJ, Parisi AF. Reproducibility of echocardiography: a study evaluating the variability of hocoung ments. Chest 1981;79:29-32.

8 Geiser EA, Conetta DA, Limacher MC, Stockton VO, Oliver LH, Jones B. A second generation computerbased edge detection algorythm for short-axis, twodimensional echocardiographic images: accuracy and improvement in interobserver variability. $\mathcal{F} \mathrm{Am}$ Soc Echo 1990;3:79-90.

9 Perez JE, Waggoner AD, Barzilai B, Melton HE, Miller JG, Sobel BE. On-line assessment of ventricular function by automatic boundary detection and ultrasonic backscatter imaging. ₹ Am Coll Cardiol 1992;19:313-20.

10 Sharma MK, Kieso RA, Fleagle SR, Marinelli C, Levett JM, Melton HE, Skorton DJ, Kerber RE, Vandenberg BF. Real-time, on-line echocardiographic measurement of $\mathrm{LV}$ function using an automated border detection of LV function using an automated (sorder detection

11 Wyatt HL, Meerbaum SL, Heng MK, Gueret P, Corday E. Cross-sectional echocardiography III. Analysis of mathematic models for quantifying volume of symmetric and asymmetric left ventricles. Am Heart $\mathcal{F} 1980$ 100:821-8.

12 Goresan J III, Schulman DS, Koch L, Thornton J, Follansbee WP. Echocardiographic automated borde detection derived left ventricular ejection fraction: comparison with radionuclide angiography [abstract]. Circulation 1991;84 (suppl II):II-585.

13 Pinto FJ, Siegel LC, Kreitzman TR, Davidson R, Popp $\mathrm{RL}$, Schnittger I. On-line estimation of cardiac output with a new automated edge detection system using transesophageal echocardiography: comparison with thermodilution [abstract]. Circulation 1991;84 (suppl II):II-585.

14 Spirito P, Maron BJ. Doppler echocardiography for assessing left ventricular diastolic function. Ann Intern Med 1988;109:122-6.
15 Snider AR. Prediction of intracardiac pressures and assessment of ventricular function with Doppler assessment of ventricular function with Dopple

16 Masuyama T, St Goar FG, Alderman ED, Popp RL Relation of mitral flow velocity patterns to left ventricular diastolic function: new insights from a combined hemodynamic and Doppler echocardiographic study. $f$ Am Coll Cardiol 1988;12:426-39.

17 Choong CY, Abascal VM, Thomas JD, Guerrero JL McGlew S, Weyman AE. Combined influence of ventricular loading and relaxation on the transmitral flow velocity profile in dogs measured by Doppler echocardiography. Circulation 1988;78:672-83.

18 Bonow RO. Radionuclide angiographic evaluation of left ventricular diastolic function. Circulation 1991;84(suppl I):I208-15.

19 Marcus ML, Weiss RM. Evaluation of cardiac structure and function with ultrafast computed tomography. In: Marcus ML, ed. Cardiac Imaging: a companion to Braunwald's

20 Klein SC, Waggoner AD, Holland MR, Miller JG, Melton HE, Perez JE. Echocardiographic on-line measurement and display of left ventricular (LV) cavity areas and function: reproducibility and normal values in control subjects [abstract]. Circulation 1991;84 (supp II):II-584.

21 Spirito P, Maron BJ, Bonow RO. Noninvasive assessment of left ventricular diastolic function: comparative analysis of Doppler echocardiographic and radionuclide angiographic techniques. $尹$ Am Coll Cardiol 1986;7: angiograph

22 Green MV, Findley SL, Bonow RO, Bacharach SL. The amount of early left ventricular filling in resting normal subjects and patients with coronary artery disease and normal systolic function. In: Ripley K, ed. Computers in Cardiology. Long Beach, CA: IEEE Computer Society, 1984:215-7.

23 Bashore TM, Leithe ME, Shaffer P. Diastolic function In: Gerson MC, ed. Cardiac Nuclear Medicine. New York: McGraw Hill, 1991:208-9.

24 Foley AD, Seward JB, Taijk AJ. Assessment of left ventricular diastolic function with a new automated echocardiographic border detection system: comparison with Doppler [abstract]. $₹ \mathrm{Am}$ Coll Cardiol 1992;19 with Doppler

25 Yellin EL, Meisner JS, Nikolic SD, Keren G. The scientific basis for the relations between pulsed-Doppler transmitral velocity patterns and left heart chambe properties. Echocardiography 1992;9:313-38. 